Running head: ATTENTION BIAS DURING EMOTIONAL MEMORY RETRIEVAL

APA copyright notice: This article may not exactly replicate the final version published in the APA journal. It is not the copy of record. http://www.apa.org/pubs/journals/emo/

Interactions between emotional attention, encoding, and retrieval of ambiguous

information: An eye-tracking study

Jonas Everaert and Ernst H. W. Koster

Ghent University, Belgium

Corresponding author:

Jonas Everaert, Ghent University, Department of Experimental Clinical and Health Psychology, Henri Dunantlaan 2, 9000 Ghent, Belgium, Phone: +32 926494 42, E-mail:

jonas.everaert@ugent.be 


\begin{abstract}
Emotional biases in attention modulate encoding of emotional material into long-term memory, but little is known about the role of such attentional biases during emotional memory retrieval. The present study investigated how emotional biases in memory are related to attentional allocation during retrieval. Forty-nine individuals encoded emotionally positive and negative meanings derived from ambiguous information, and then searched their memory for encoded meanings in response to a set of retrieval cues. The remember/know/new procedure was used to classify memories as recollection- or familiarity-based, and gaze behavior was monitored throughout the task to measure attentional allocation. We found that a bias in sustained attention during recollection-based, but not familiarity-based, retrieval predicted subsequent memory bias toward positive vs. negative material following encoding. Thus, during emotional memory retrieval, attention affects controlled forms of retrieval (i.e., recollection), but does not modulate relatively automatic, familiarity-based retrieval. These findings enhance understanding of how distinct components of attention regulate the emotional content of memories. Implications for theoretical models and emotion regulation are discussed.
\end{abstract}

Keywords: attention, memory, retrieval, recollection, familiarity 


\section{Interactions between emotional attention, encoding, and retrieval of ambiguous information: An eye-tracking study}

Attention and memory are components of human cognition intricately related to emotional well-being. Healthy individuals, for example, allocate more attention to positive compared to negative stimuli and show better memory for positive than negative events (Gotlib \& Joormann, 2010). Emotional biases in attention and memory have conventionally been studied as separate topics such that the interactions between these processes are poorly understood (Everaert, Koster, \& Derakshan, 2012). Despite recent advances in knowledge on how attention at encoding regulates emotional long-term memory (e.g., Everaert, Duyck, \& Koster, 2014; Talmi \& McGarry, 2012), little is known about the role of attention during emotional memory retrieval.

Prior research on attentional influences on retrieval of emotional material produced mixed results. One study using a concurrent task to divide attention observed that the emotion-enhanced memory effect was not influenced by divided attention at retrieval (ClarkFoos \& Marsh, 2008), indicating that retrieval of emotional material can occur successfully in the absence of focused attention. However, another study reported that the enhancement effect disappeared with divided attention (Maddox, Naveh-Benjamin, Old, \& Kilb, 2012), suggesting that emotional memory retrieval is an attentionally-demanding process. Similarly, basic cognitive studies applying a divided attention paradigm has also yielded inconsistent findings on the role of attention during recall of neutral material (e.g., Fernandes \& Moscovitch, 2000; Naveh-Benjamin, Kilb, \& Fisher, 2006).

The extent to which attention during retrieval affects memory may depend on the retrieval process, namely recollection vs. familiarity (Dudukovic, DuBrow, \& Wagner, 2009). Recollection (i.e., retrieval of specific details about the prior occurrence of an item) and familiarity (i.e., a sense of having encountered an item without retrieving specific details) rely 
on at least partly different cognitive and neural mechanisms (Yonelinas, Aly, Wang, \& Koen, 2010). The former is attentionally-demanding while the latter can be largely automatic (Jacoby, 1991). Basic research suggest that divided attention during retrieval reduces memory for recollected items but leaves familiarity-based retrieval intact (Hicks \& Marsh, 2000).

The distinction between recollection- and familiarity-based retrieval may also be key in emotional memory retrieval: Controlled and automatic processes may differentially recruit emotional attention to guide memory search and retrieval. In support of this idea, studies have shown that emotional content (e.g., Ochsner, 2000) and affective states (e.g., Jermann, Van der Linden, Laurencon, \& Schmitt, 2009) influence retrieval by boosting recollection. However, no prior research has tested how attention is deployed toward retrieval cues that are used to guide memory search, and how this relates to the retrieved memory.

The goal of the present study was to examine attention biases toward positive vs. negative retrieval cues that are used to guide memory search in an eye-tracking task enabling assessment of attentional allocation to retrieval cues during recollection- and familiarity-based retrieval. It was hypothesized that: (1) attention bias during familiarity-based retrieval will not be related to familiarity memory bias, while attention bias during recollection-based retrieval will be related to recollection memory bias; (2) sustained attention (i.e., assessed by fixation durations on retrieval cues) and not attentional selection (i.e., assessed by the number of fixations on retrieval cues) bias during recollection-based retrieval is related to recollection memory bias; and (3) sustained attention bias during recollection-based retrieval predicts recollection memory bias when controlling for encoding bias. 


\section{Method}

\section{Participants}

Forty-nine undergraduate students (45 women; age: 18 - 32 years) were recruited. All participants were native Dutch speakers with normal or corrected-to-normal vision. Participants were paid 10 euro.

\section{Stimuli}

Encoding task. A set of 30 emotional and 20 neutral scrambled sentences was drawn from Everaert et al. (2014). The positive and negative target words presented in each emotional scrambled sentence (e.g., "winner" and "loser" in "am winner born loser a I") were matched on word class, word length $\left(M_{\text {positive }}=8.50, S D_{\text {positive }}=2.24, M_{\text {negative }}=8.50\right.$, $\left.S D_{\text {negative }}=1.91 ; t<1, p>.05\right)$, and word frequency (log frequency per million; $M_{\text {positive }}=1.14$, $\left.S D_{\text {positive }}=0.65, M_{\text {negative }}=1.13, S D_{\text {negative }}=0.50 ; t<1, p>.05\right)$ using WordGen $($ Duyck, Desmet, Verbeke, \& Brysbaert, 2004). Word position within each scrambled sentence was randomized with the constraint that emotional words did not occur next to each other, nor as a first or last word within a scrambled sentence. This controls for parafoveal processing of adjoining words and wrap-up effects (Staub \& Rayner, 2007). The positive word was presented before the negative word in $50 \%$ of the emotional scrambled sentences. Word order criteria imposed on emotional scrambled sentences were also applied to target words in neutral scrambled sentences (e.g., "cinema" and "theatre" in "the I theatre visit cinema often"). All scrambled sentences were self-referent and six words long.

Memory task. The 30 emotional and 20 neutral target word pairs of the scrambled sentences from encoding served as retrieval cues in the memory task. These 'old' items were mixed with an equal number of 'new' emotional and neutral distractor word pairs retrieved from Everaert et al. (2014). The 'new' emotional target words pairs were matched on word class, word length $\left(M_{\text {positive }}=8.03, S D_{\text {positive }}=2.08, M_{\text {negative }}=7.97, S D_{\text {negative }}=1.71 ; t<1, p>.05\right)$, 
and word frequency $\left(\log\right.$ frequency per million; $M_{\text {positive }}=1.33, S D_{\text {positive }}=0.74, M_{\text {negative }}=1.14$, $\left.S D_{\text {negative }}=0.62 ; t=1.03, p=.31\right)$. There were no differences between 'old' and 'new' negative and positive target words $(p-\mathrm{s}>.05)$.

\section{Procedure}

Figure 1 depicts the trial events of each experimental task.

Encoding task. Participants started with a computerized version of the scrambled sentences test (SST) modeled after Everaert et al. (2014). On each trial, a neutral or emotional scrambled sentence was displayed following fixation (left-aligned to elicit left-to-right reading). While the item was on-screen, participants were instructed to unscramble the sentence into its two semantically different meanings using five of the six words (e.g., "am winner born loser a I" into "I am a born winner" and "I am a born loser"), to elaborate thoroughly on how each statement applied to them, and select the most self-relevant option. In contrast to standard task instructions (cf. Everaert et al., 2014), here the instructions encouraged encoding of both sentences with one statement serving as the target cognition in the memory test. The task automatically continued after 30s or when participants pressed the spacebar. The next trial display presented the scrambled sentence with each word numbered prompting participants to verbally report the sentence selected using the numbers.

The complete task displayed emotional and neutral scrambled sentences in fixed random order, divided over 5 blocks of each 6 emotional and 4 neutral sentences. No more than two emotional scrambled sentences were consecutively presented to minimize priming effects. ${ }^{1}$ As in prior research, a cognitive load procedure was added to each block to reduce social desirable responses: A six digit number had to be memorized before and recalled after a block (Rude, Wenzlaff, Gibbs, Vane, \& Whitney, 2002). ${ }^{2}$

Retention interval. After encoding, participants worked for 3 minutes on a digitsymbol substitution test of the Wechsler Adult Intelligence Scale $3^{\text {th }}$ edition (Wechsler, 1997). 
Memory task. Next, participants engaged in an incidental memory test. Here, each trial presented two cue words at the same eccentricity $\left(5^{\circ}\right)$ above or below central fixation. Cues were target words from the SST (e.g., "winner" and "loser" on test trials) or new words (e.g., "smart" and "stupid" on distractor trials). The on-screen position of positive and negative cues was counterbalanced. Test and distractor trials were presented in random order.

While the cues were on-screen, participants were instructed to use the cues to search their memory to determine if they constructed sentences with the words during the previous task and to recall the sentence selected if they thought to have used them before. Participants continued by pressing the spacebar to describe their retrieval experience. Using a remember/know/new paradigm (Tulving, 1985) following recommendations by Migo, Mayes, and Montaldi (2012), participants judged if their retrieval was based on recollection vs. familiarity, or whether the word pair was new using following options: "Type A" (i.e., Remember: the word pair cued the recall of details of the sentence selected), "Type B" (i.e., Know: a feeling that the word pair was previously encountered without recalling associated details from the prior exposure), or "New". Next, participants specified the retrieved sentence (for Type A) or selected the cue that triggered the highest sense of familiarity (for Type B). Participants were trained prior to the task to ensure correct understanding of the distinct retrieval experiences.

Attentional bias. Eye movements were recorded when scrambled sentences (encoding task) or retrieval cues (retrieval task) were on-screen to measure attention toward positive and negative words during encoding and retrieval, respectively. This methodology enables online assessment of attention while participants elaborate on self-relevant meanings and use cues to guide memory search. 


\section{Eye tracking}

Experimental tasks were coded in SR Research Experiment Builder and eye movements were registered with a tower-mounted Eyelink 1000 device (SR Research, Mississauga, Ontario, Canada). Viewing was binocular and eye movements were recorded from the right eye. Fixations were sampled every millisecond and considered when longer than 50ms. Areas of interest were the negative and positive target/cue words in the encoding/memory task.

\section{Cognitive bias indices}

Attention bias indices reflecting processing of positive over negative material were computed for following variables: (1) attentional selection at encoding; (2) sustained attention at encoding; (3) attentional selection during familiarity-based retrieval; (4) attentional selection during recollection-based retrieval; (5) sustained attention during familiarity-based retrieval; (6) sustained attention during recollection-based retrieval. Bias indices, calculated within-subjects, were based on number of fixations and fixation durations on positive and negative words during the encoding and memory tasks (see also Armstrong \& Olatunji, 2012). The number of fixations on positive words was divided by the total number of fixations on positive and negative words to index attentional selection bias. Analogous calculations led to a relative bias index for fixation duration to index sustained attention bias. These relative bias indices control for inter-individual baseline fixation differences due to inter-individual variability in reading performance.

Encoding bias was indexed by the ratio of selected positively unscrambled sentences over the total selected positive and negative sentences. Memory bias indices were computed based on the dual-process signal detection model of recognition conceiving recollection as a threshold process and familiarity as a signal detection process (Yonelinas et al., 2010). The proportion of positive items out of all hits and false alarms was calculated separately for 
Remember (recollection) and Know (familiarity) reports as well as the proportion of misses and correct rejections (see Table 1). Following Yonelinas et al. (2010), a recollection memory bias index was calculated by subtracting the proportion of false remember (i.e., false alarms, misses) from correct remember (i.e., hits, correct rejections) responses. For familiarity memory bias, $d$ ' was computed by subtracting $z$-transformed false alarm rates from $z$ transformed hit rates. Higher scores reflect a greater ability to discriminate encoded positive from distractor positive items relative to negative items.

\section{Results}

\section{Associations between cognitive biases}

Correlations between bias indices were inspected per retrieval process to test associations between attention bias during retrieval and memory bias. Table 2 presents descriptive statistics and correlation coefficients. In line with hypotheses 1 and 2 , the correlations between familiarity memory bias and each of the indices of attention bias during familiarity-based retrieval were not significantly different from zero. Regarding recollectionbased retrieval, the correlation between memory bias and sustained attention bias was significantly different from zero, while the correlation between memory bias and attentional selection was not. The correlations between memory bias and the attention bias indexes were not significantly different for both familiarity-based $(z=0.65, p=.52)$ and recollection-based $(z=0.78, p=.43)$ processes.

Note that attentional selection bias during encoding was not significantly different from $50 \%, t(48)=1.42, p=.16$, indicating positive and negative words were equally fixated. This suggests compliance with task instructions to consider both the positively and negatively unscrambled statements. 


\section{Does attentional bias at retrieval regulate recollection of encoded material?}

Hypothesis 3 was tested via a two-step hierarchical regression analysis on recollection memory bias scores with encoding bias and sustained attention bias during recollection-based retrieval entered in the first and second step, respectively. All predictors were z-transformed and the dependent variable showed no substantial deviations from normality (skewedness=0.57, kurtosis $=-2.15)$. Collinearity statistics were within acceptable limits indicating low levels of multicollinearity (VIF's $<1.08$, Tolerance's $>.93$ ). Table 3 presents the statistical models tested. The results showed that encoding bias did not add to the model, $F<1, p=.34$. In line with the hypothesis, adding sustained attention bias scores in a second step significantly contributed to the model, explaining $8.9 \%$ of the variance in recollection memory bias, $\Delta F(1,46)=4.58, p<.05$. The variables included in step 2 accounted in total for $10.8 \%$ of the variance, with sustained attention bias $(\beta=.31, p<.05)$ but not encoding bias $(\beta=.06, p=.71)$ as a significant predictor.

\section{Discussion}

This study observed that attention bias during recollection-based retrieval predicts accurate recollection of emotional material after controlling for encoding bias. This suggests that retrieval of emotional memories benefits from strategic allocation of attention toward appropriate retrieval cues during memory search. Interestingly, not attentional selection but sustained attention toward positive vs. negative retrieval cues may regulate controlled emotional memory retrieval. That is, successful recollection memory depends on how long one focuses attention on a particular cue and not on attentional operations that select cues to guide memory search. Whereas attention may play a role in controlled, recollection-based retrieval, the current findings also indicate that attention toward retrieval cues does not modulate automatic, familiarity-based retrieval. Specifically, the results showed that the 
correlations between attentional biases at retrieval and familiarity memory bias were not significantly different from zero.

The findings are consistent with prior research indicating that recollection- and familiarity-based processes differentially recruit attention to guide memory search (Hicks \& Marsh, 2000) and may explain earlier contrasting findings supporting either automatic (ClarkFoos \& Marsh, 2008) or attention-demanding (Maddox et al., 2012) nature of emotional memory retrieval. Moreover, the observations extend prior research on attention biases during encoding in accounting for emotional memory (Talmi \& McGarry, 2012) by showing that attention bias also regulates memory during controlled but not automatic retrieval. Sustained attention biases may regulate emotional memory during both encoding (Everaert et al., 2014) and retrieval stages.

The findings have implications for our understanding of the mechanisms underlying emotion regulation strategies. When people are exposed to ambiguous, stressful situations (e.g., a job interview) with competing cues (e.g., a frowning versus a smiling interviewer), one may focus attention on a particular cue to guide memory search in an attempt to retrieve a prior experience (e.g., "last time I did well on an interview: I'm a winner"). Depending on the cue attended and the memory retrieved, one may repair or worsen the distressed mood.

Several limitations should be noted. First, the high number of female participants limits the generalizability of the results to men. Second, measurement of attention via eyetracking informs on overt attention and provides limited insight into covert attentional shifts. Finally, the study design forces the use of relative bias indices that compare the processing of positive vs. negative material. This is because the emotional scrambled sentences have a different semantic context than the neutral scrambled sentences, which makes it difficult to compare eye movements across emotional and neutral sentences. Here, neutral scrambled sentences were included to control for affective priming effects between emotional scrambled 
sentences. This approach cannot determine whether the observed effects were driven by a greater emphasis on positive material or a lack of responsiveness to negative material.

In conclusion, this study revealed that the influence of attention during emotional memory retrieval depends on the retrieval process and the attentional operation. Future research may further map out how attention during retrieval regulate what is remembered. 


\section{Footnotes}

${ }^{1}$ Neutral scrambled sentences served as control stimuli, and data from these sentences are not considered here.

${ }^{2}$ Performance on the cognitive load test was satisfactory for all participants and all blocks of trials were included in the analyses. 


\section{Acknowledgements}

The authors thank Mariam Aly for her thoughtful comments on an earlier version of this manuscript. 


\section{References}

Armstrong, T., \& Olatunji, B. O. (2012). Eye tracking of attention in the affective disorders: A meta-analytic review and synthesis. Clinical Psychology Review, 32(8), 707-723. doi: 10.1016/j.cpr.2012.09.004

Clark-Foos, A., \& Marsh, R. L. (2008). Recognition memory for valenced and arousing materials under conditions of divided attention. Memory, 16(5), 530-537. doi: $10.1080 / 09658210802007493$

Dudukovic, N. M., DuBrow, S., \& Wagner, A. D. (2009). Attention during memory retrieval enhances future remembering. Memory \& Cognition, 37(7), 953-961. doi: 10.3758/Mc.37.7.953

Duyck, W., Desmet, T., Verbeke, L. P. C., \& Brysbaert, M. (2004). WordGen: A tool for word selection and nonword generation in Dutch, English, German, and French. Behavior Research Methods Instruments \& Computers, 36(3), 488-499. doi: 10.3758/BF03195595

Everaert, J., Duyck, W., \& Koster, E. H. W. (2014). Attention, interpretation, and memory biases in subclinical depression: A proof-of-principle test of the combined cognitive biases hypothesis. Emotion, 14(2), 331-340. doi: 10.1037/a0035250

Everaert, J., Koster, E. H. W., \& Derakshan, N. (2012). The combined cognitive bias hypothesis in depression. Clinical Psychology Review, 32(5), 413-424. doi: 10.1016/j.cpr.2012.04.003

Fernandes, M. A., \& Moscovitch, M. (2000). Divided attention and memory: Evidence of substantial interference effects at retrieval and encoding. Journal of Experimental Psychology-General, 129(2), 155-176. doi: 10.1037/0096-3445.129.2.155 
Gotlib, I. H., \& Joormann, J. (2010). Cognition and depression: Current status and future directions. Annual Review of Clinical Psychology, 6, 285-312. doi: 10.1146/annurev.clinpsy.121208.131305

Hicks, J. L., \& Marsh, R. L. (2000). Toward specifying the attentional demands of recognition memory. Journal of Experimental Psychology: Learning, Memory, and Cognition, 26(6), 1483-1498. doi: 10.1037//0278-7393.26.6.1483

Jacoby, L. L. (1991). A Process Dissociation Framework - Separating Automatic from Intentional Uses of Memory. Journal of Memory and Language, 30(5), 513-541. doi: $10.1016 / 0749-596 x(91) 90025-\mathrm{F}$

Jermann, F., Van der Linden, M., Laurencon, M., \& Schmitt, B. (2009). Recollective experience during recognition of emotional words in clinical depression. Journal of Psychopathology and Behavioral Assessment, 31(1), 27-35. doi: 10.1007/s10862-0089093-1

Maddox, G. B., Naveh-Benjamin, M., Old, S., \& Kilb, A. (2012). The role of attention in the associative binding of emotionally arousing words. Psychonomic Bulletin \& Review, 19(6), 1128-1134. doi: 10.3758/s13423-012-0315-x

Migo, E. M., Mayes, A. R., \& Montaldi, D. (2012). Measuring recollection and familiarity: Improving the remember/know procedure. Consciousness and Cognition, 21(3), 14351455. doi: 10.1016/j.concog.2012.04.014

Naveh-Benjamin, M., Kilb, A., \& Fisher, T. (2006). Concurrent task effects on memory encoding and retrieval: Further support for an asymmetry. Memory \& Cognition, 34(1), 90-101. doi: 10.3758/Bf03193389

Ochsner, K. N. (2000). Are affective events richly recollected or simply familiar? The experience and process of recognizing feelings past. Journal of Experimental Psychology-General, 129(2), 242-261. doi: 10.1037//0096-3445.129.2.242 
Rude, S. S., Wenzlaff, R. M., Gibbs, B., Vane, J., \& Whitney, T. (2002). Negative processing biases predict subsequent depressive symptoms. Cognition \& Emotion, 16(3), 423440. doi: 10.1080/02699930143000554

Staub, A., \& Rayner, K. (2007). Eye movements and on-line comprehension processes. In M. G. Gaskell (Ed.), The Oxford Handbook of Psycholinguistics. Oxford: Oxford University Press.

Talmi, D., \& McGarry, L. M. (2012). Accounting for immediate emotional memory enhancement. Journal of Memory and Language, 66(1), 93-108. doi: 10.1016/j.jml.2011.07.009

Tulving, E. (1985). Memory and Consciousness. Canadian Psychology-Psychologie Canadienne, 26(1), 1-12. doi: 10.1037/H0080017

Wechsler, D. (1997). Manual for the Wechsler Adult Intelligence Scale - Third Edition. San Antonio, TX: Harcourt Assessment.

Yonelinas, A. P., Aly, M., Wang, W. C., \& Koen, J. D. (2010). Recollection and familiarity: Examining controversial assumptions and new directions. Hippocampus, 20(11), 1178-1194. doi: 10.1002/Hipo.20864 
Table 1. Memory performance

\begin{tabular}{|c|c|c|c|c|}
\hline & \multicolumn{2}{|c|}{ Negative items } & \multicolumn{2}{|c|}{ Positive items } \\
\hline & Remember & Know & Remember & Know \\
\hline Hits & $2.65(2.31)$ & $0.86(1.78)$ & $8.96(3.72)$ & $6.57(4.15)$ \\
\hline False alarms & $0.02(0.14)$ & $0.47(1.00)$ & $0.31(0.51)$ & $2.08(2.23)$ \\
\hline Misses & \multicolumn{2}{|c|}{$1.04(1.47)$} & \multicolumn{2}{|c|}{$4.94(2.25)$} \\
\hline
\end{tabular}


Table 2. Correlations between cognitive bias indices.

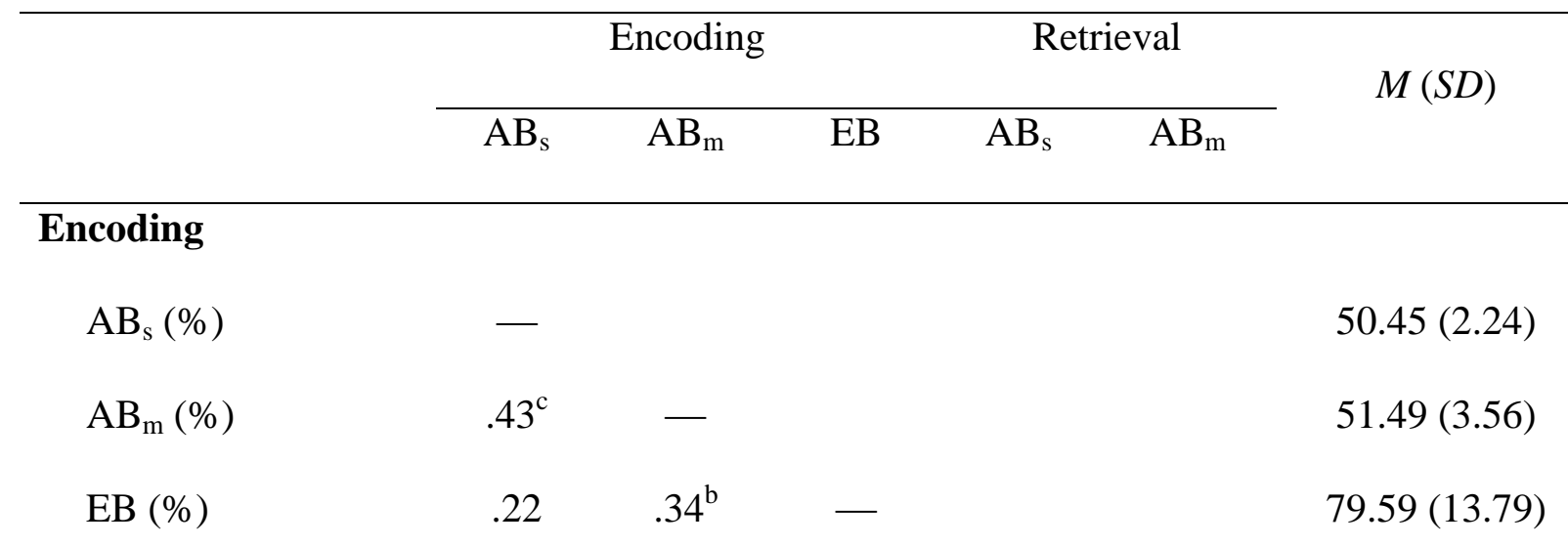

Familiarity

$\begin{array}{lllllll}\mathrm{AB}_{\mathrm{s}}(\%) & .32^{\mathrm{b}} & .23 & .07 & - & & 51.14(5.87) \\ \mathrm{AB}_{\mathrm{m}}(\%) & .27^{\mathrm{a}} & .14 & -.03 & .73^{\mathrm{d}} & - & 52.26(7.34) \\ \mathrm{MB}\left(\mathrm{d}^{\prime}\right) & -.24 & -.03 & -.25^{\mathrm{a}} & -.05 & .02 & 0.00(1.35)\end{array}$

Recollection

$\begin{array}{lcccccc}\mathrm{AB}_{\mathrm{s}}(\%) & .24 & -.10 & .02 & - & & 54.50(6.34) \\ \mathrm{AB}_{\mathrm{m}}(\%) & .07 & -.09 & .25^{\mathrm{a}} & .67^{\mathrm{d}} & - & 55.27(8.33) \\ \mathrm{MB} \text { (threshold) } & .18 & -.22 & .14 & .22 & .31^{\mathrm{b}} & 43.87(38.84)\end{array}$

Notel. Spearman's rank correlation coefficients are displayed. Note $2 .{ }^{\mathrm{a}} p<.10,{ }^{\mathrm{b}} p<.05,{ }^{\mathrm{c}} p<.01$, ${ }_{p}^{\mathrm{d}}<.001$. Note $3 . \mathrm{AB}_{\mathrm{s}}=$ attention selection bias, $\mathrm{AB}_{\mathrm{m}}=$ attention maintenance bias, $\mathrm{EB}=$ encoding bias, $\mathrm{MB}=$ memory bias. 
Table 3. Hierarchical regression model predicting recollection bias.

\begin{tabular}{llrrrrrr}
\hline Predictor & $b$ & $S E_{b}$ & $\beta$ & $t$ & \multicolumn{1}{c}{$\Delta R^{2}$} \\
& & & & & & \\
\hline Step 1 & Constant & 0.48 & 0.10 & & $4.83^{\mathrm{d}}$ & .019 \\
& Encoding bias & 0.00 & 0.00 & .14 & 0.96 & \\
& & & & & & \\
Step 2 & Constant & -0.12 & 0.30 & & -0.39 & $.089^{\mathrm{b}}$ \\
& Encoding bias & 0.00 & 0.00 & .06 & 0.38 & \\
& Sustained attention during retrieval & 0.01 & 0.01 & .31 & $2.14^{\mathrm{b}}$ & \\
& & & & & & \\
\end{tabular}

Note. ${ }^{\mathrm{a}} p<.10,{ }^{\mathrm{b}} p<.05,{ }^{\mathrm{c}} p<.01,{ }^{\mathrm{d}} p<.001$. 


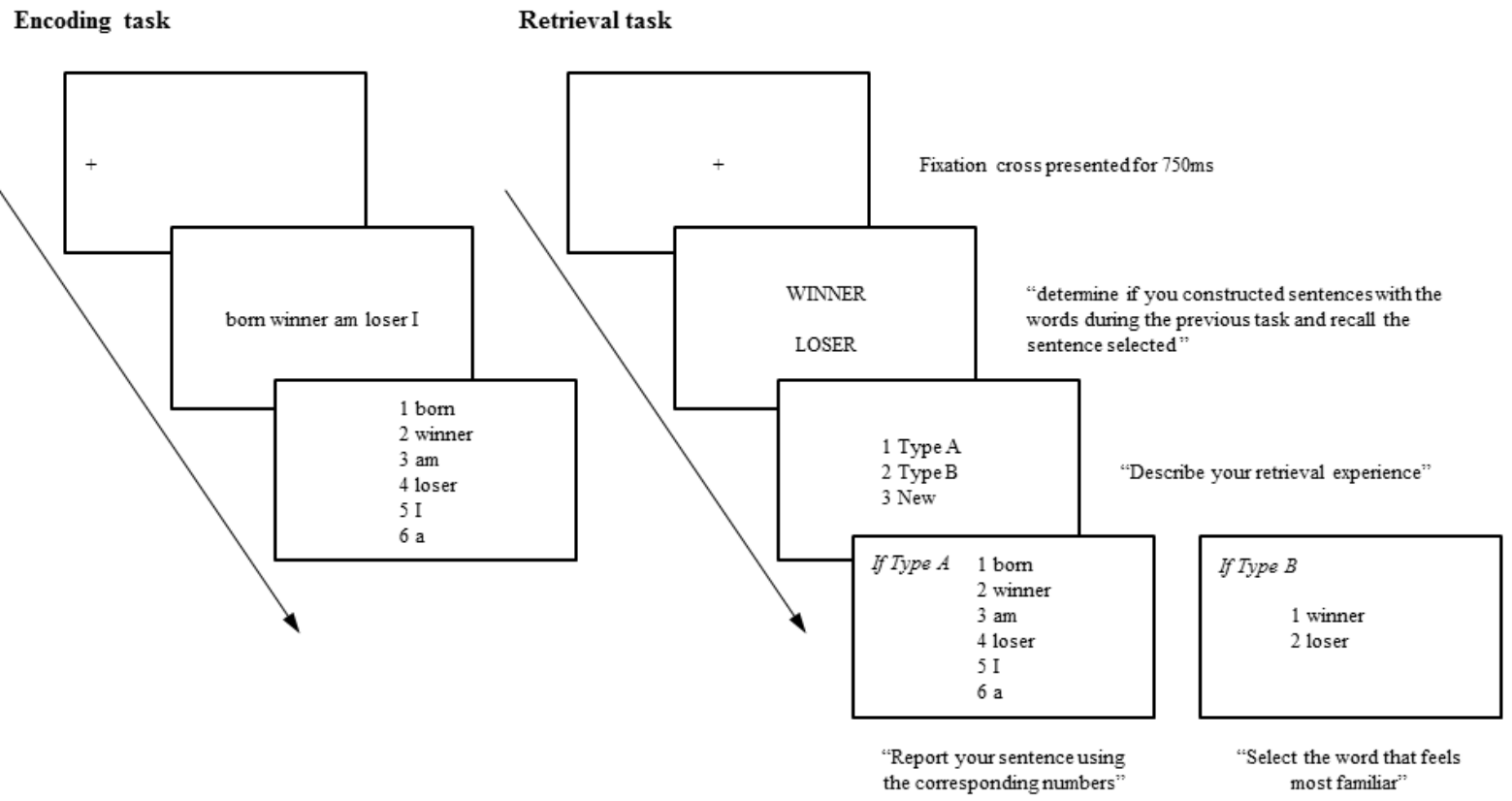

Figure 1. Example of the flow of trial events on the encoding and memory task. 